\title{
Ultra-high-molecular-weight polyethylene sublaminar tape as semirigid fixation or pedicle screw augmentation to prevent failure in long-segment spine surgery: an ex vivo biomechanical study
}

\author{
Remco J. P. Doodkorte, MSc, ${ }^{1}$ Ricardo Belda, PhD, ${ }^{2}$ Alex K. Roth, PhD, ${ }^{1}$ \\ Bert van Rietbergen, PhD, ${ }^{1,3}$ Jacobus J. Arts, PhD, ${ }^{1,3}$ L. M. Arno Lataster, MSc, MEd, ${ }^{4}$ \\ Lodewijk W. van Rhijn, MD, PhD, ${ }^{1}$ and Paul C. Willems, MD, PhD'
}

\begin{abstract}
1Department of Orthopaedic Surgery, Research School CAPHRI, Maastricht University Medical Center, Maastricht, The Netherlands; ${ }^{2}$ Centre of Research in Mechanical Engineering-CIIM, Department of Mechanical Engineering and Materials, Universitat Politècnica de València, Spain; ${ }^{3}$ Orthopaedic Biomechanics, Department of Biomedical Engineering, Eindhoven University of Technology, Eindhoven; and ${ }^{4}$ Department of Anatomy and Embryology, Faculty of Health, Medicine and Life Sciences, Maastricht University, Maastricht, The Netherlands
\end{abstract}

OBJECTIVE Complications after adult spinal deformity surgery are common, with implant-related complications occurring in up to $27.8 \%$ of cases. Sublaminar wire fixation strength is less affected by decreasing trabecular bone density in comparison to pedicle screw (PS) fixation due to the predominant cortical bone composition of the lamina. Sublaminar fixation may thus aid in decreasing implant-related complications. The goal of this study was to compare fixation characteristics of titanium sublaminar cables (SCs), ultra-high-molecular-weight polyethylene (UHMWPE) tape, PSs, and PSs augmented with UHMWPE tape in an ex vivo flexion-bending setup.

METHODS Thirty-six human cadaver vertebrae were stratified into 4 different fixation groups: UHMWPE sublaminar tape (ST), PS, metal SC, and PS augmented with ST (PS + ST). Individual vertebrae were embedded in resin, and a flexion-bending moment was applied that closely resembles the in vivo loading pattern at transitional levels of spinal instrumentation.

RESULTS The failure strength of PS + ST $(4522 \pm 2314 \mathrm{~N})$ was significantly higher compared to the SC $(2931 \pm 751 \mathrm{~N})$ and PS $(2678 \pm 827 \mathrm{~N})$ groups, which had $p$ values of 0.028 and 0.015 , respectively (all values expressed as the mean $\pm \mathrm{SD}$ ). Construct stiffness was significantly higher for the PS groups compared to the stand-alone sublaminar wiring groups $(p=0.020)$. In contrast to SC, ST did not show any case of cortical breach.

CONCLUSIONS The higher failure strength of PS + ST compared to PS indicates that PS augmentation with ST may be an effective measure to reduce the incidence of screw pullout, even in osteoporotic vertebrae. Moreover, the lower stiffness of sublaminar fixation techniques and the absence of damage to the cortices in the ST group suggest that ST as a stand-alone fixation technique in adult spinal deformity surgery may also be clinically feasible and offer clinical benefits. https://thejns.org/doi/abs/10.3171/2020.6.SPINE20605

KEYWORDS sublaminar wires; pedicle screws; biomechanics; spinal deformity; surgical technique

A DULT spinal deformity (ASD) is increasingly prevalent, with asymptomatic incidences reported to be as high as $68 \%$ in adults older than 60 years. ${ }^{1}$ Surgical spinal fusion may be indicated for patients with severe imbalance or neurological symptoms, or in patients not responding to conservative treatment. ${ }^{2}$ The annual number of spinal fusion procedures for ASD has increased by $273 \%$ between 2001 and 2013 in the National (Nationwide) Inpatient Sample of the US. ${ }^{3}$

Pedicle screws (PSs) provide a rigid connection between the vertebrae and the spinal rods. However, PS constructs are susceptible to screw pullout or junctional failure, especially in bone with diminished bone quality, such as in patients with osteoporosis. ${ }^{4,5}$ The revision rate due

ABBREVIATIONS ASD = adult spinal deformity; BMD = bone mineral density; FE = finite element; $\mathrm{PS}=$ pedicle screw; $\mathrm{SC}=$ sublaminar cable; $\mathrm{ST}=$ sublaminar tape; UHMWPE = ultra-high-molecular-weight polyethylene.

SUBMITTED April 16, 2020. ACCEPTED June 17, 2020

INCLUDE WHEN CITING Published online October 30, 2020; DOI: 10.3171/2020.6.SPINE20605. 

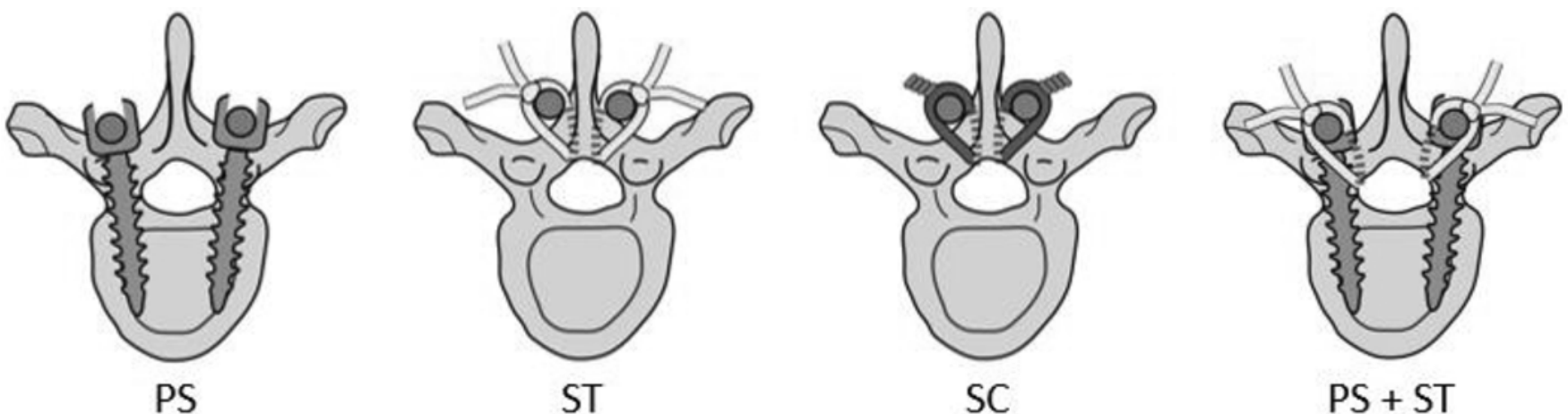

FIG. 1. Schematic illustration of the experimental study groups. The vertebrae are instrumented bilaterally with PSs, UHMWPE ST, titanium SCs, or PSs augmented with UHMWPE ST (i.e., PS + ST). Figure is available in color online only.

to implant failure after ASD correction is $27.8 \%$ within 2 years of clinical follow-up. ${ }^{6}$ Sublaminar wire fixation has been shown to be less dependent on bone mineral density (BMD) compared to PSs, ${ }^{7}$ because the trabecular bone within the pedicle and the vertebral body are mainly affected in osteoporosis. PS fixation augmented with sublaminar wires may thus potentially decrease the incidence of PS pullout.

Metal sublaminar wires have been frequently associated with complications, such as laminar cut-in and cable failure. ${ }^{8}$ Alternatively, polymer tape has been introduced to address these disadvantages. ${ }^{9,10}$ The wide profile of the sublaminar tape (ST) increases the contact area, resulting in lower peak stresses. Hence, higher correction forces can be applied. Furthermore, ultra-high-molecular-weight polyethylene (UHMWPE) ST has a substantially higher fatigue strength in comparison to metal sublaminar wires. ${ }^{11}$

The goal of this ex vivo biomechanical study was to assess the instantaneous failure characteristics of different spinal fixation methods by using human cadaveric vertebrae in a flexion-bending setup to represent loads occurring at the ends of a long-segment construct. We hypothesize that sublaminar fixation possesses a lower fixation stiffness and an equal or higher pullout strength compared to PSs. Furthermore, augmenting PSs with ST is expected to significantly increase the failure load.

PS fixation strength is generally assessed ex vivo by using an axial pullout test setup, whereas the fixation strength of sublaminar wires has been assessed using a wide variety of testing methods. ${ }^{12-16}$ Only a few studies have directly compared different fixation techniques by using an experimental setup that mimics in vivo loading of construct ends..$^{17,18}$ In this study, in vivo loading conditions due to a flexion movement were simulated. Bilateral spinal rods were used as a cantilever beam, leveraging around a fixed fulcrum point, ultimately applying in a force and moment to different spinal fixators. Using this setup, metal sublaminar wires were compared to UHMWPE ST and PSs. Additionally, the potential benefit of augmenting PSs with UHMWPE ST is assessed. A finite element (FE) model representing the experimental setup was then used to quantify loads, and to investigate differences in load distribution and stress magnitudes within the vertebrae between PS and ST configurations.

\section{Methods \\ Specimens}

Six fresh-frozen human thoracolumbar spinal columns were obtained from the Department of Anatomy and Embryology, Maastricht University. A handwritten and signed codicil was received from all donors. Specimens were stored at $-30^{\circ} \mathrm{C}$ and thawed in tap water before further manipulation. Plain radiographs were made of the specimens to exclude vertebrae with fractures or metastatic disease. Dual energy x-ray absorptiometry (DEXA) scans were made to determine BMD. Subsequently, the vertebral columns were separated into individual vertebrae and denuded of soft tissue. After pilot testing, use of spine segments for another experiment, and exclusion of fractured and metastatic vertebrae, 36 vertebrae remained for biomechanical testing.

\section{Embedding}

All specimens were embedded in polymethylmethacrylate (PMMA, Technovit 3040; Heraeus Kulzer) with the superior endplate positioned vertically and the base of the pedicles completely embedded. To allow for sublaminar wire passage after embedding, the posterior half of the spinal canal was kept free from embedding material by using molding clay.

\section{Instrumentation}

The specimens were stratified over the following instrumentation groups (Fig. 1) based on level and BMD: PSs, ST, sublaminar cable (SC), and ST-augmented PSs (i.e., PS + ST).

\section{Pedicle Screws}

Monoaxial PSs (CD Horizon; Medtronic Sofamor Danek) were placed bilaterally by an experienced orthopedic spine surgeon (P.C.W.) before embedding. The entry points were identified using anatomical landmarks and subsequently breached using a drill and bone awl. The pedicles were prepared under visual guidance with a pedicle probe. Next, care was taken to insert the PSs parallel to the superior endplate. After placing the spinal rods, the set screws were fixed with $9 \mathrm{Nm}$ of torque in accordance with the manufacturer's recommendations. 

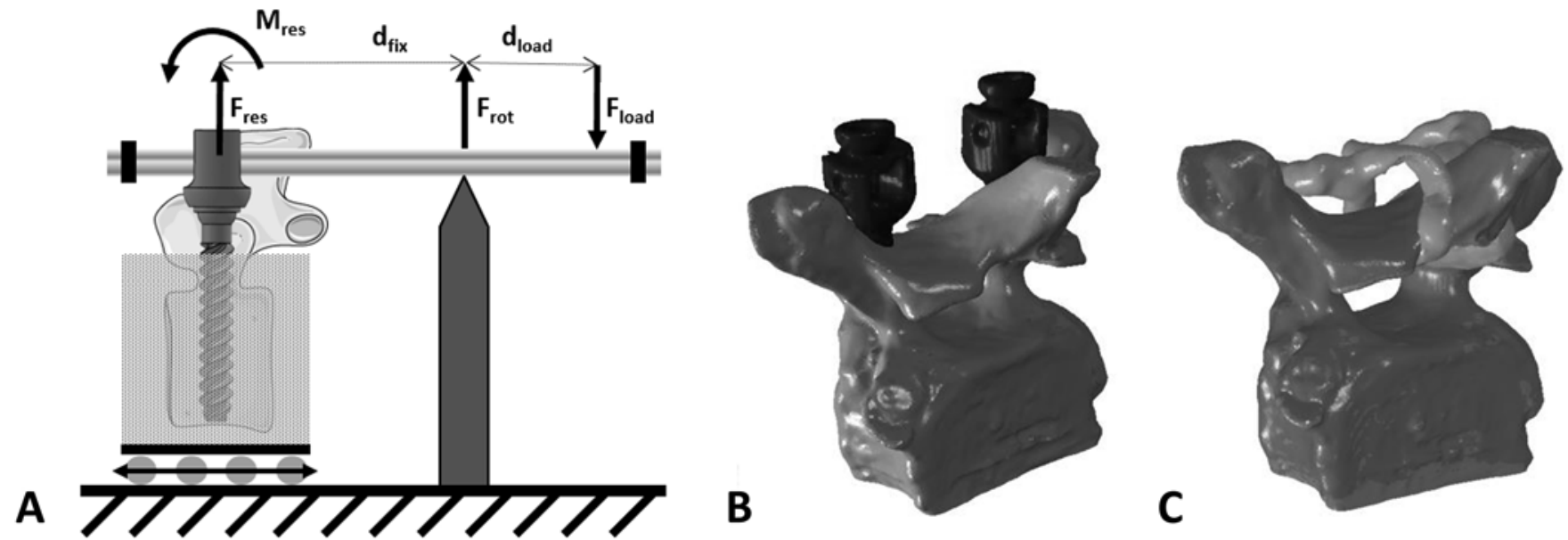

FIG. 2. A: Each vertebra was embedded with the PS trajectory in a vertical position. Bilateral spinal rods were stabilized by 2 cross-links (black rectangles) and positioned over a fulcrum point $\left(F_{\text {rot }}\right)$. The applied load $\left(F_{\text {load }}\right)$ resulted in a flexion movement and a resistant force $\left(F_{\text {res }}\right)$ by the vertebral fixation. In the case of PS fixation, a moment is applied around the head of the PS $\left(M_{\text {res }}\right)$ The distance between fixation and fulcrum point $\left(d_{f i x}\right)$, and the distance between fulcrum point and load application $\left(d_{\text {load }}\right)$ were kept constant between the experiments at 75 and $25 \mathrm{~mm}$, respectively. B and C: The FE meshes developed to evaluate PS (B) and ST (C) fixation constructs. Boundary conditions were applied to the FE meshes to replicate experimental tests for the analysis of load distribution within the vertebrae. Figure is available in color online only.

\section{Sublaminar Tape}

The ST was passed through the spinal canal around the lamina and knotted using a Klemheist knot with 2 wraps. ${ }^{19}$ The UHMWPE ST is a 4-mm-wide radiopaque woven tape made with Dyneema Purity Radiopaque fibers. ${ }^{11}$ After a first half-hitch, the knot was tensioned up to $500 \mathrm{~N}$ with a custom-made device. Finally, the knots were secured with 6 additional reversed half-hitches on alternating posts. ${ }^{20}$

\section{Sublaminar Cable}

The titanium SCs $(01.0 \mathrm{~mm})$ (Atlas cable system; Medtronic Sofamor Danek) were instrumented analogous to the ST. Tensioning (156 N) and fastening was performed according to the manufacturer's recommendations.

\section{Sublaminar Tape-Augmented Pedicle Screws}

The specimen was instrumented with PSs, as described above. Subsequently, the ST was passed through the spinal canal and knotted, tensioned up to $500 \mathrm{~N}$, and secured as described before.

\section{Pullout Testing}

The embedded vertebrae were mounted on a sliding carriage with sleeve bearings oriented with the caudal end toward the fulcrum point (Fig. 2) to represent loading at the superior end of a construct. Subsequently, 10-mm custom-made stainless steel rods, tapered at the end to fit the 5.5-mm PSs, were placed over a fulcrum and connected to the vertebrae according to the designated fixation members (PS, ST, SC, or PS + ST). The rods were cross-linked at the cranial as well as the caudal end. In case of sublaminar fixation, the cross-links were fixed before tensioning the cables/tapes. The distance between the fulcrum and the screw insertion point $(75 \mathrm{~mm})$, and the distance between loading rod and fulcrum $(25 \mathrm{~mm})$ were kept constant for all samples.

After a preload phase up to $50 \mathrm{~N}$ at $20 \mathrm{~mm} /$ minute, the construct was loaded at $10 \mathrm{~mm} /$ minute by using a universal testing machine (Instron E3000; Instron). Load was increased until construct failure occurred. Data were analyzed above $100 \mathrm{~N}$ of applied load to allow for setting of the construct. Stiffness of the construct was defined as the slope of the force-displacement curve between $100 \mathrm{~N}$ and $500 \mathrm{~N}$, and is expressed in Newtons per millimeter $(\mathrm{N} / \mathrm{mm})$. The mechanism of failure was noted.

\section{Statistical Analysis}

The failure load was defined as the peak load and extracted from the force-displacement data. One-way ANOVA was performed to test for differences across groups for age, BMD, failure load, and stiffness. Pooled stiffnesses were compared by an independent samples ttest. Vertebral level stratification over the different groups was assessed using a Kruskal-Wallis test. Statistical significance was set at $\mathrm{p}<0.05$ for all statistical analyses.

\section{FE Modeling}

An FE model of a single human vertebra (male, 96 years old, T9 level) instrumented with PSs and ST was developed to replicate the experiment in silico. The vertebra was scanned by microCT (microCT100; SCANCO Medical) at a 34.2- $\mu \mathrm{m}$ spatial resolution $(70 \mathrm{kV}, 200 \mu \mathrm{A}, 300 \mathrm{msec}$, Al $0.5-\mathrm{mm}$ filter), and was subsequently downscaled to clinical CT resolution $(0.5 \mathrm{~mm})$. Initially, the vertebra was scanned without instrumentation, and subsequently with ST and finally with PSs. A custom-made device was used to replicate the orientation of the vertebrae in the scanner. This allowed for overlaying of the noninstrumented and instrumented segmented images, allowing for accurate seg- 
TABLE 1. Demographics of donors of the vertebrae included in the analysis

\begin{tabular}{|c|c|c|c|c|c|c|c|c|c|c|c|c|c|c|c|c|}
\hline \multirow[b]{2}{*}{ Group (no.) } & \multirow[b]{2}{*}{ Age, Yrs } & \multirow[b]{2}{*}{$\mathrm{BMD}, \mathrm{g} / \mathrm{cm}^{2}$} & \multicolumn{14}{|c|}{ Vertebral Levels } \\
\hline & & & T3 & $\mathrm{T} 4$ & T5 & T6 & $\mathrm{T} 7$ & T8 & T9 & $\mathrm{T} 10$ & $\mathrm{~T} 11$ & $\mathrm{~T} 12$ & L1 & L2 & L3 & L4 \\
\hline PS (8) & $87.00 \pm 5.56$ & $0.640 \pm 0.209$ & & 2 & 1 & 1 & & 1 & & & 1 & 1 & & & & 1 \\
\hline ST (9) & $77.89 \pm 9.41$ & $0.610 \pm 0.154$ & & 1 & 1 & & 2 & 1 & & 1 & & 1 & & 1 & 1 & \\
\hline SC (9) & $77.89 \pm 9.41$ & $0.703 \pm 0.211$ & 1 & 1 & 1 & 1 & 1 & 1 & & 1 & & & 1 & 1 & & \\
\hline PS + ST (8) & $77.89 \pm 9.41$ & $0.622 \pm 0.252$ & 1 & 1 & 1 & 2 & & 1 & & & & & & 1 & 1 & \\
\hline
\end{tabular}

Values for age and BMD are expressed as the mean \pm SD.

mentation. The downscaled images of the vertebra were segmented using ScanIP (Simpleware), and FE models of linear tetrahedral meshes were generated using Abaqus (version 6.14, Dassault Systèmes) (Fig. 2B and C). Bone elements were assigned mineral content $(\rho)$-dependent material properties, considering tissues $\rho$ with from 0.004 to $1.085 \mathrm{gHA} / \mathrm{cm}^{3}$. A power law relationship $\left(\mathrm{E}(\rho)=4.73 \rho^{1.56}\right.$ $\mathrm{GPa}$ ) reported in the literature for vertebral cancellous bone was taken into account, ${ }^{21}$ and a Poisson's ratio of 0.3 was defined for bone tissue. Isotropic elastic properties were considered for PS material $(\mathrm{E}=110 \mathrm{GPa}, v=0.3)$. The ST was modeled as an isotropic elastic material (UHMWPE: $\mathrm{E}=5 \mathrm{GPa}, v=0.3^{11}$ ), whereas the bilateral spinal rods were modeled as rigid connector elements. Linear elastic simulations were carried out and a load of $1000 \mathrm{~N}$ was applied to the rods bilaterally at a distance $\left(d_{\text {load }}\right)$ of $25 \mathrm{~mm}$ from the rotation point. At the other end, the rod was rigidly attached to the screw head or wire knot at a distance $\left(d_{f i x}\right)$ of $75 \mathrm{~mm}$. Similar to the experiment, only free displacement parallel to the rods was allowed (Fig. 2A). From the FE model, the axial reaction force $\left(F_{r e s}\right)$ was obtained and the applied bending moment $\left(M_{r e s}\right)$ at the screw/wire then followed from the bending moment equilibrium equation (Fig. 2A): $F_{\text {res }} d_{f i x}+M_{\text {res }}-F_{\text {load }} d_{\text {load }}=0$ (Equation 1).

\section{Results}

\section{Specimen Demographics}

Two samples from the PS group were excluded due to early pedicle fracture during testing. Thus, a total of 34 vertebrae (of the 36 available specimen) were included in the analysis. Allocation of the vertebrae to the 4 different groups resulted in nonsignificant differences for age $(\mathrm{p}=$ $0.103)$, BMD ( $p=0.785)$, and vertebral level $(p=0.743)$ (Table 1).

\section{Ex Vivo Biomechanical Test}

A significantly higher failure load was measured for the $\mathrm{PS}+\mathrm{ST}$ group $(4522 \pm 2314 \mathrm{~N})$ in comparison to the PS group $(2678 \pm 827 \mathrm{~N})(\mathrm{p}=0.015)$, and the SC group (2931 $\pm 751 \mathrm{~N})(\mathrm{p}=0.028)$. No statistically significant difference was found between the PS + ST and ST $(3563 \pm 1428 \mathrm{~N})$ group $(\mathrm{p}=0.170)$. No statistically significant differences were measured between the PS, ST, and SC groups (Fig. 3 , Table 2).

The stiffness of the PS and PS + ST groups were comparable, with mean stiffness of PS $(794 \pm 168 \mathrm{~N} / \mathrm{mm})$ and $\mathrm{PS}+\mathrm{ST}(802 \pm 237 \mathrm{~N} / \mathrm{mm})$ respectively, $(\mathrm{p}=0.929)$. The stiffness of the ST and SC groups were also comparable
$(637 \pm 134 \mathrm{~N} / \mathrm{mm}$ and $653 \pm 172 \mathrm{~N} / \mathrm{mm}$, respectively; $\mathrm{p}$ $=0.855)$. Statistical comparison of individual groups did not render statistically significant differences. However, a pooled comparison between PS-based fixations (PS and $\mathrm{PS}+\mathrm{ST})(798 \pm 205 \mathrm{~N} / \mathrm{mm})$ compared to both sublaminar fixation techniques $(645 \pm 150 \mathrm{~N} / \mathrm{mm})$ was significant $(\mathrm{p}$ $=0.020$ ).

Distinct differences in failure mode were observed between the 4 groups, as is shown in Fig. 4 . In both the PS and PS + ST groups, all but one of the samples failed by screw pullout. One sample failed by pedicle fracture in both groups. In the ST group, one sample failed by lamina fracture, whereas the remainder failed by pedicle fracture. Two samples failed by wire breakage in the SC group. Testing of the SC specimen resulted in either unilateral or bilateral cortical breach.

All of the load-displacement curves showed a similar initial pattern with a toe region up to approximately 100 $\mathrm{N}$ followed by a near linear region until failure (Fig. 5). In the PS group, the steep linear increase continued until the yield point, where either a single or a double failure pattern could be identified, depending on the occurrence of simultaneous or consecutive PS failure. Discordantly, the SC group showed a short linear region, followed by a stochastic load-displacement increase until failure. Finally, the PS + ST group had a loading phase consisting of 2 separate linear parts. After an initial lower stiffness, a distinct region with a higher stiffness can be discerned.

\section{FE Simulations}

In the PS construct, $1000 \mathrm{~N}$ of loading resulted in an $\mathrm{F}_{\text {res }}$ of $312 \mathrm{~N}$. From the bending equation (Equation 1), an $\mathrm{M}_{\text {res }}$ of $1.6 \mathrm{Nm}$ follows. For the ST case very similar values were found.

In the PS construct, a maximum principal stress of \pm 9 $\mathrm{MPa}$ was found at the pedicles. In agreement with the failure patterns in the ex vivo biomechanical tests, the screw thread and the pedicles endure the highest stress (Fig. 6AC). The ST fixation simulation resulted in distinctly different load distribution compared to PS fixation. The forces of the ST acting on the laminae predominantly distribute through the cortical bone of the laminae and the pedicles (Fig. 6D-F). At $1000 \mathrm{~N}$ applied load, a maximum principal stress of $\pm 17 \mathrm{MPa}$ is predicted in the cortex of the pedicles.

\section{Discussion}

In this ex vivo biomechanical study simulating the loading pattern at proximal ends of spinal constructs, the 

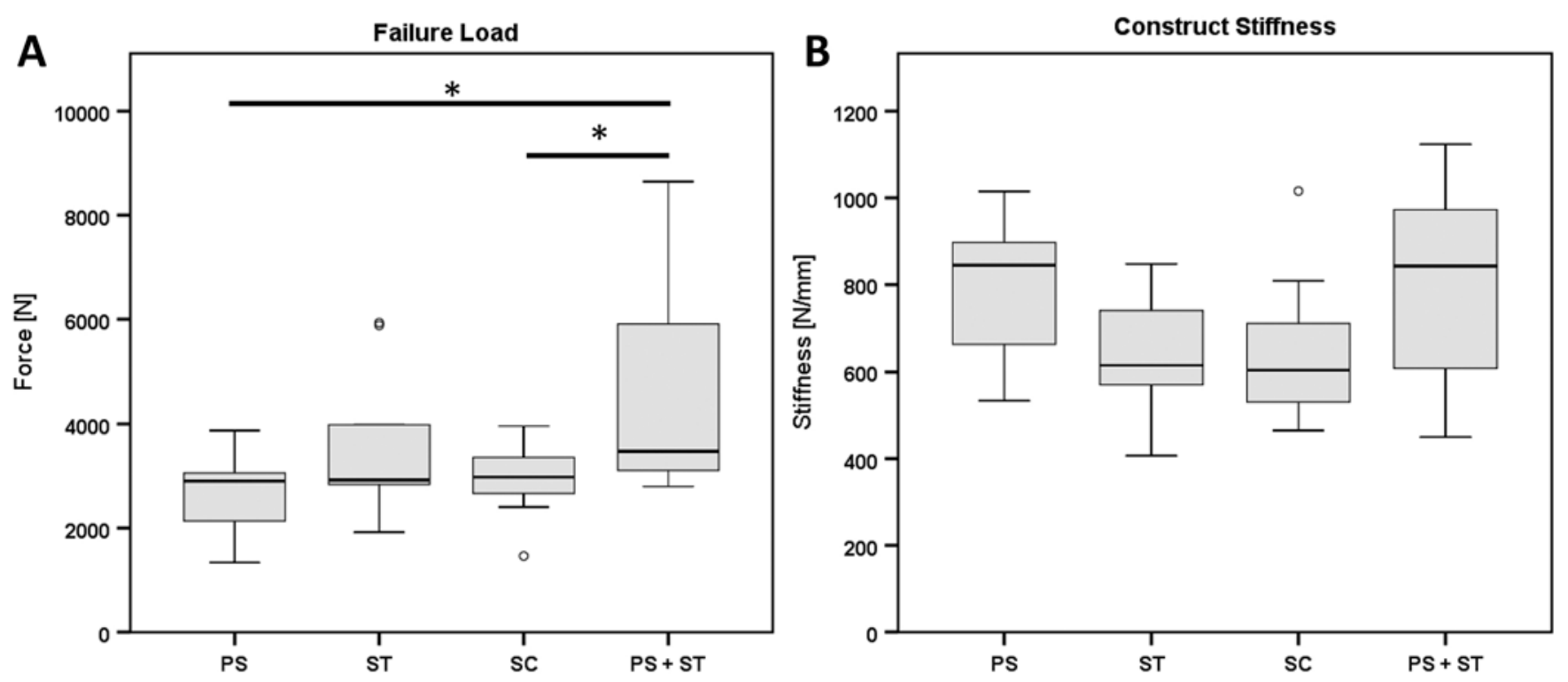

FIG. 3. Boxplots of failure load and construct stiffness. Boxes and whiskers represent interquartile ranges and significance is indicated by asterisks. A: The applied force at failure was comparable between the PS, ST, and SC construct groups. The PS + ST group displayed a significantly higher failure load compared to the PS group. B: A clear distinction can be observed between stand-alone SC/ST groups and PS-based groups: PS and PS + ST constructs had significantly higher construct stiffness compared to sublaminar constructs (ST and SC). No significant differences were found between SC and ST. * $p<0.05$.

instantaneous failure characteristics of different spinal fixation methods were studied. A flexion-bending moment was applied until construct failure, to allow for direct comparison between PSs, SCs, ST, and PS + ST in the same test setup with clinically relevant failure modes for all groups. The PS + ST group had a significantly higher failure load compared to PS, whereas both types of sublaminar fixation (ST and SC) resulted in a significantly lower construct stiffness. The observed failure modes demonstrate the improved force distribution attained with ST compared to SC, with no laminar cut-in occurring in the ST group.

The PS + ST group displayed a higher ultimate failure strength compared to PS. This is in agreement with Sun et al., who demonstrated similar differences in a bending setup with calf spinal segments, indicating that PS + ST at the distal ends of long-segment spinal constructs provides mechanical benefit. ${ }^{18}$ No differences in failure strengths for SC, ST, and PS were found in the current study. This is in agreement with Paxinos et al., who compared the fixation strength between PS and sublaminar wire fixation in human vertebrae with low BMD. ${ }^{16}$ Several studies have found lower mean ultimate failure strength magnitudes for the SC and ST groups. ${ }^{12,18}$ This could be explained by the considerably lower BMD in these studies (0.190-0.360 $\mathrm{g} / \mathrm{cm}^{2}$, compared with $0.400-1.106 \mathrm{~g} / \mathrm{cm}^{2}$ in the current study). Considering the higher BMD and the positive linear relationship between PS fixation strength and BMD in our study, the pullout strength of PSs is expected to have been relatively high.,16

Although failure loads were similar, the construct stiffness was higher for PS constructs compared to SC and ST constructs. This is in agreement with the findings of Sun et al., and confirms the semirigid nature of sublaminar fixation. The reduced construct stiffness of sublaminar fixation hints at the possibility to use it as a means to achieve topping off, with the aim to avoid proximal junction

TABLE 2. Failure loads and stiffness (mean $\pm S D$ ) with pairwise $p$ values

\begin{tabular}{ccccc}
\hline Group & PS & ST & SC & PS + ST \\
\hline Failure load (N) & $2678 \pm 827$ & $3563 \pm 1428$ & $2931 \pm 751$ & $4522 \pm 2314$ \\
\hline PS & - & 0.219 & 0.722 & $0.015^{*}$ \\
\hline ST & - & - & 0.362 & 0.17 \\
\hline SC & - & - & - & $0.028^{*}$ \\
\hline Stiffness (N/mm) & $794 \pm 168$ & $637 \pm 134$ & $653 \pm 172$ & $802 \pm 237$ \\
\hline PS & - & 0.083 & 0.117 & 0.929 \\
\hline ST & - & - & 0.855 & 0.069 \\
\hline SC & - & - & - & 0.098 \\
\hline *Indicates significance with $p<0.05$ & & & &
\end{tabular}



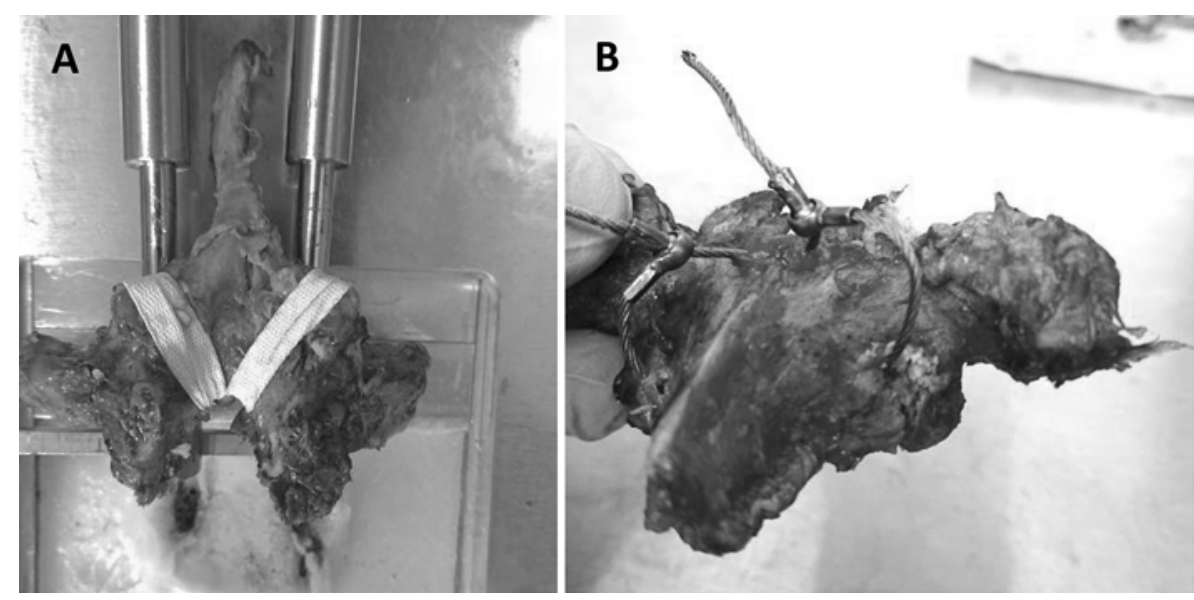

\begin{tabular}{lllll} 
C & PS & ST & SC & PS \\
& $(8)$ & $(9)$ & $(9)$ & $\begin{array}{l}\text { ST } \\
(8)\end{array}$ \\
\hline $\begin{array}{l}\text { Screw } \\
\text { pullout }\end{array}$ & 7 & 0 & 0 & 7 \\
$\begin{array}{l}\text { Pedicle } \\
\text { fracture }\end{array}$ & 1 & 8 & 7 & 1
\end{tabular}

FIG. 4. A: The ST has a wide $(4 \mathrm{~mm})$ profile and therefore creates a larger contact area compared to titanium SC. This resulted in the absence of macroscopically detectable lamina damage. B: Flexion-bending testing resulted in laminar cortical breach in each of the specimens. C: Failure mode comparison between the 4 instrumentation groups. Figure is available in color online only.

kyphosis. ${ }^{22}$ Recently, a number of studies have analyzed these possibilities both biomechanically and clinically for clamp-based sublaminar systems. ${ }^{23-25}$ The low fatigue strength of metal cables does not allow for stand-alone fixation at construct ends, and thus the use of UHMWPE tape may offer great benefit. Therefore, thorough biomechanical comparisons in human cadaver thoracolumbar spine segments should be performed to elucidate this additional potential benefit.

Comparing the PS + ST group to the PS group, a similar stiffness was found. As mentioned previously, the loaddisplacement curve of the PS + ST group showed a 2-stage progression; initially, there is a linear region when load is predominantly carried by the PSs, and secondly, an increasingly steep linear region at higher loads. The cables do not encompass the rods at the exact same position at which the screws are attached. Thus, presumably the ST only supported the screws when the rods started to deform elastically at higher forces. Because the ST augmentation does not increase construct stiffness, it may reduce PS pullout without increasing the risk of proximal junctional kyphosis in long-segment instrumented spinal fusion. ${ }^{26}$ Clinical evaluation will ultimately be needed to elucidate the effect of ST augmentation on clinical complication rates.

No significant differences were found between the SC and ST groups in terms of stiffness and ultimate failure strength. The majority of specimens failed by pedicle base fracture in both groups, indicating a superior strength of the lamina compared to the pedicles. In the study performed by Hongo et al., a 6-mm polyester ST was compared to a monofilament SC, with no significant differences between their ultimate failure strength either. ${ }^{12}$ However, differences in behavior are clearly reflected by the failure modes of these fixation types. Broken SCs were observed in a few cases, similar to clinical observations, whereas ST did not fail. Moreover, breaching of the cortex was observed in all SC specimens, compared to zero occurrences in the ST group. This confirms previous findings by Murakami et al., who demonstrated that a higher force is necessary to cut through the lamina for flat braided tape compared to SC. ${ }^{8}$
To our knowledge, no FE modeling studies investigating ST fixation have been published. FE modeling on spinal fixation techniques is primarily focused on PS fixation..$^{27-30}$ In our study, FE modeling provided insight into the applied bending moment and the load distribution within the vertebrae as a result of load transferred through PS and ST fixations. If no bending moment can be transferred (as in classic pullout strength tests), an axial force of $333.3 \mathrm{~N}$ would have resulted in our setup (Equation 1). However, the model resulted in a considerable bending moment because the screw/tape constrained the rotation, and this reduced the actual axial force by $6.4 \%$ to $312 \mathrm{~N}$. It should be noted, however, that because the rod was modeled as rigid, the bending moment found here is an upper-end value. It should also be noted that the attachment of the rod to the tape results in an over-constraint situation because the tape is modeled as fixed (glued) to the bone. Nevertheless, the analyses clearly indicate that the experimental setup indeed provides a different loading compared to classic pullout tests.

The highly loaded regions predicted by the models were consistent with the failure locations observed experimentally for both PS and ST configurations. As expected, the PS model presented high strain values around the screw, which relates to the screw pullout occurrences. Assuming linear behavior up to failure (Fig. 6), the average PS failure load $(2678 \mathrm{~N})$ would result in an ultimate stress of $24 \mathrm{MPa}$, which is reasonable at the transition between cortical and cancellous bone. ${ }^{31}$ For the ST case, the average failure load of $3563 \mathrm{~N}$ would translate to an ultimate stress of $61 \mathrm{MPa}$, which also in agreement with cortical bone failure values. Nonetheless, the peak stresses may be overestimated because the natural flexibility of the ST was not fully represented in the model. Peak stress distributions in the pedicles are in agreement with pedicle base fractures as the experimental primary failure mode. Improved models of the ST, and ultimately combining both fixation methods, could demonstrate optimal load distribution through both the trabecular and the cortical bone in PS + ST constructs. 
A

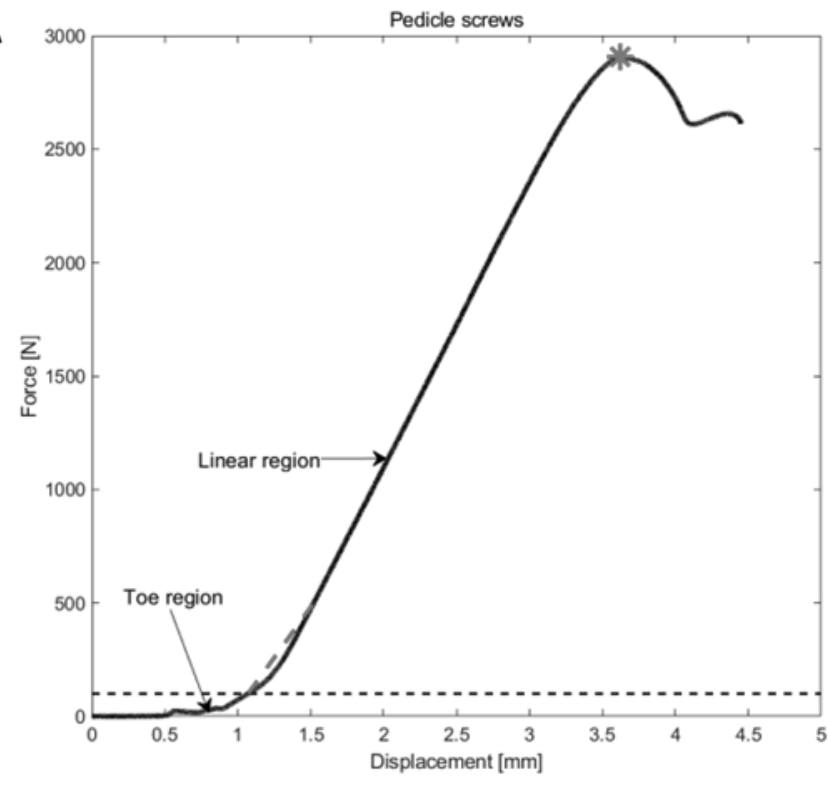

C

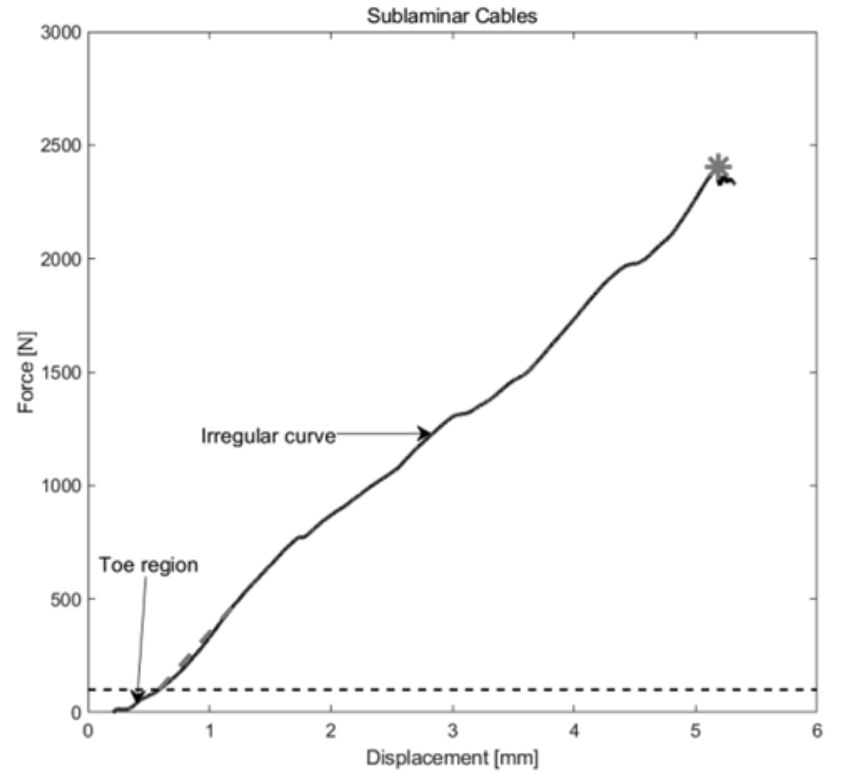

B

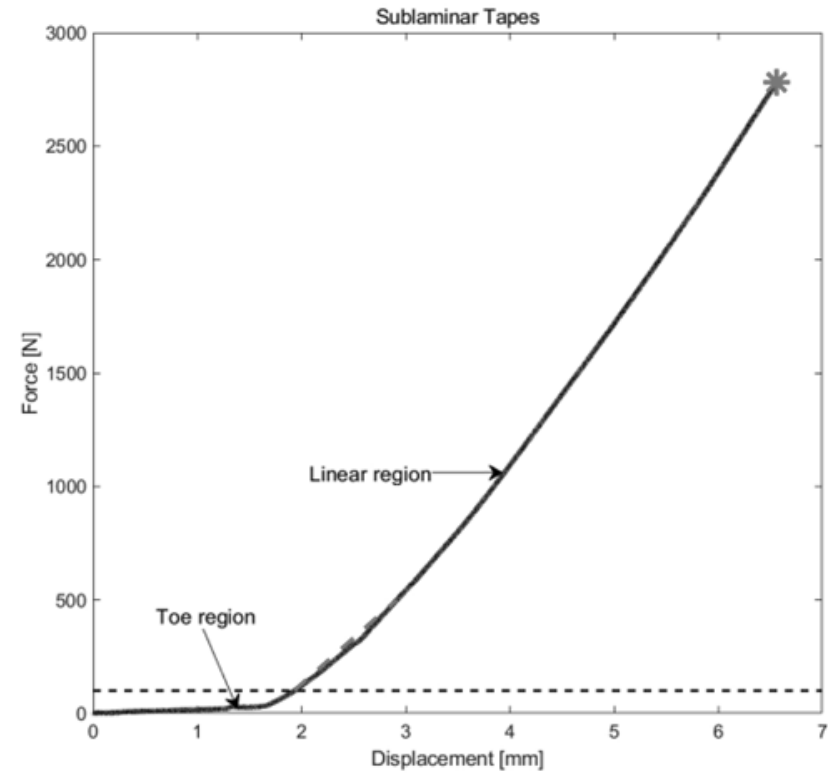

D

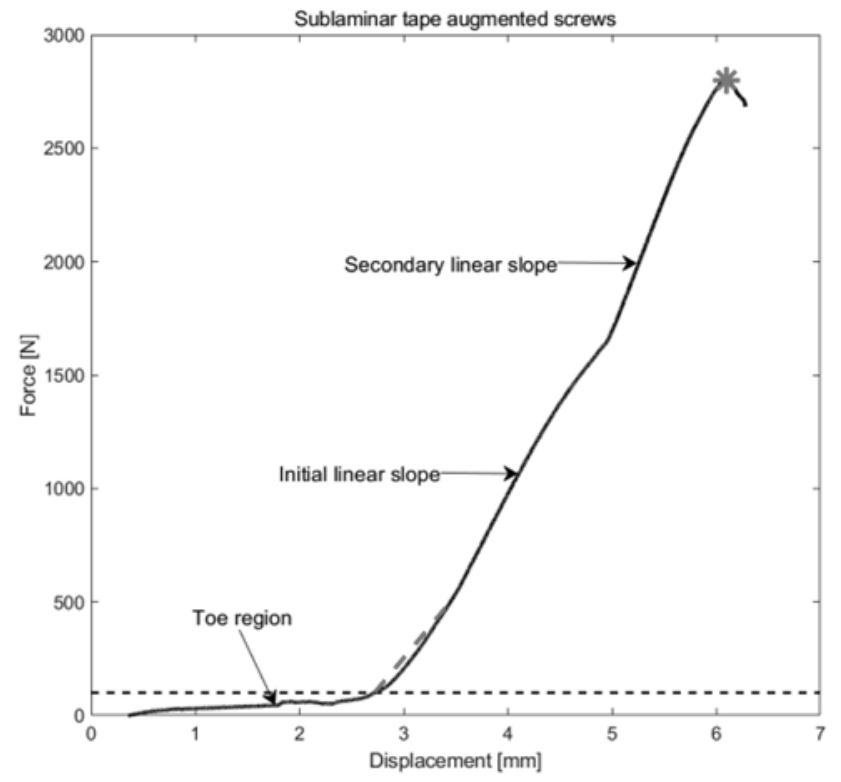

FIG. 5. Representative force-displacement curve for each of the construct types. Yield force indicated with red star and stiffness calculated over the red dotted linear slope. A: PS constructs displayed a near linear force-displacement curve. After failure, the resistance to load disappeared immediately. B: ST constructs also displayed a near linear load-displacement relationship. The resistance to force was completely released after peak force due to failure by pedicle fractures. C: SC constructs displayed an irregular curve resulting from cutting of the cable into the lamina. Force was released immediately after reaching failure load. D: Constructs with ST-augmented PSs (i.e., PS + ST) typically demonstrated 2 distinct slopes: an initial linear slope, followed by a steeper linear slope. In case the failure load resulted in PS pullout only, further resistance to load was achieved by the SCs until pedicle fracture. Figure is available in color online only.

We acknowledge certain limitations of this study. Previously, various studies have shown a relation between PS fixation strength and BMD., ${ }^{7,16,32}$ In an effort to create robust and clinically relevant data, we did not restrict the included vertebral levels. Instead, thoracic and lumbar levels were stratified over the 4 experimental groups. Due to the wide variety of included vertebrae and a limited amount of specimen, effect modification of BMD could not be properly assessed. Furthermore, it should be noted that this test setup only measures initial fixation strength of the various fixation members and cannot provide information on the long-term biomechanical differences between the methods of fixation.

\section{Conclusions}

Our study demonstrated a higher ultimate fixation strength of PS + ST compared to stand-alone PS or SC. 

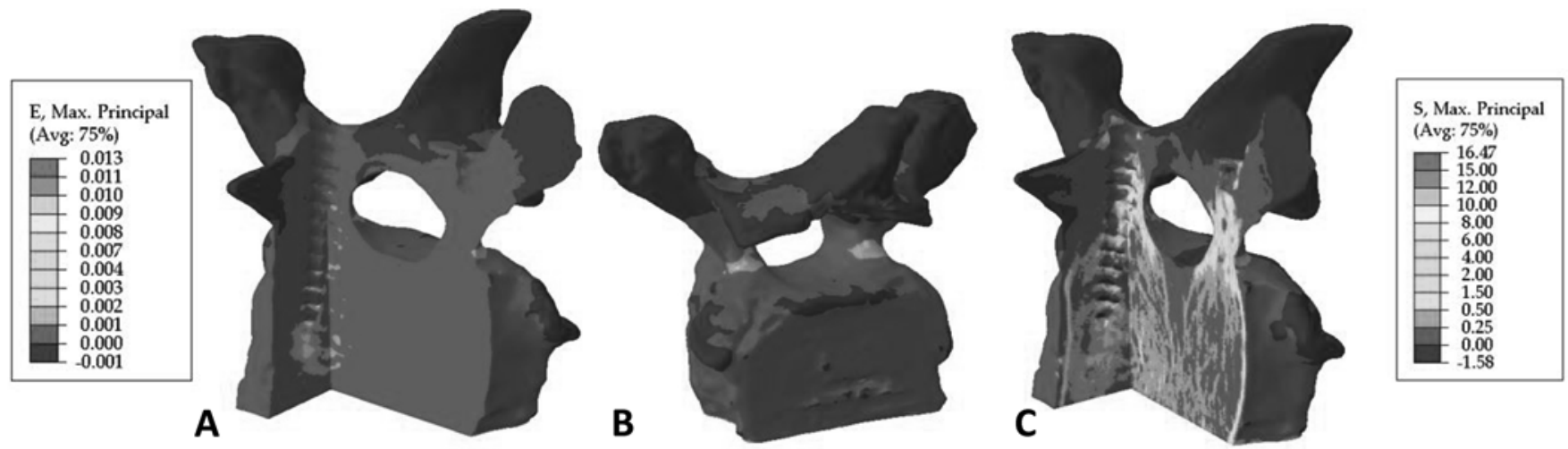

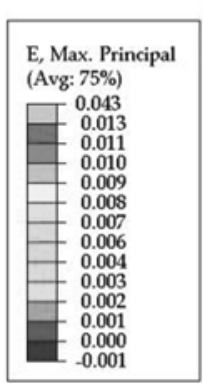

D

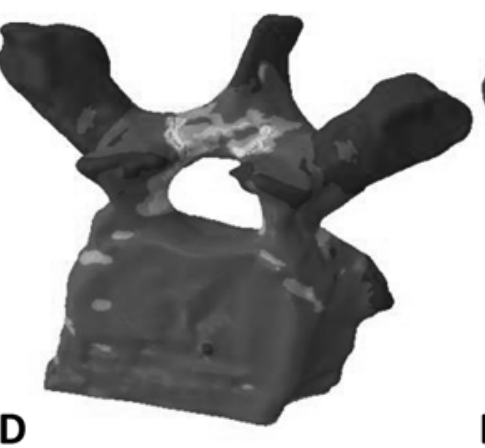

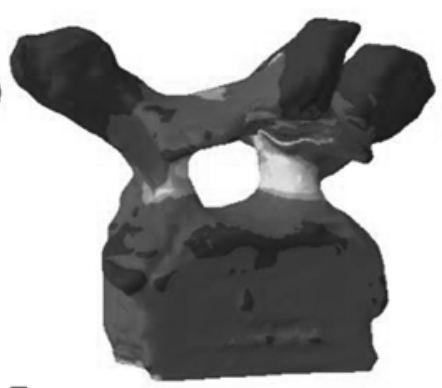

$\mathbf{E}$

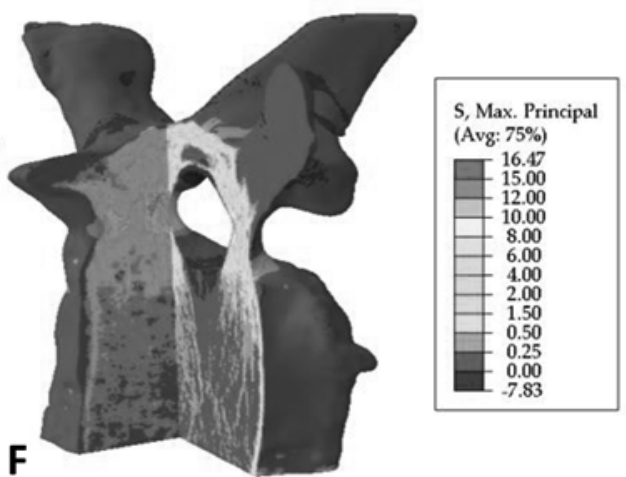

FIG. 6. Maximum principal stress and strain distributions resulting from the FE simulations with 1000-N applied load $\left(F_{\text {load }}\right)$. A-C: PSs: higher strains are present in the inner trabecular regions around the screws (A) and in the pedicles (B). Stresses are concentrated at the pedicles and around the screw (C). D-F: ST: high strain areas are consistent with the failure locations observed experimentally for ST construct. The lamina and pedicle regions suffer from high strains $(D$ and $E)$. The cortex in the lamina and the pedicles distribute the loads to the vertebral body (F). Figure is available in color online only.

This implies that PS augmentation at the ends of longsegment spinal constructs may be an effective means to avoid screw pullout. Furthermore, this study showed no instrument breakage and no laminar damage when using UHMWPE ST, whereas both issues commonly occurred with titanium SC. Additionally, sublaminar fixation overall exhibits a lower stiffness than PS fixation. Considering the low BMD of elderly patients, stand-alone application of ST in the ASD population may be clinically feasible and may offer clinical benefits.

\section{Acknowledgments}

This work has been performed under the framework of Chemelot InSciTe.

Dr. Belda is thankful for the support received by the Spanish Ministerio de Ciencia, Innovación y Universidades grant numbers DPI2013-46641-R and DPI2017- 89197-C2-2-R.

\section{References}

1. Schwab F, Dubey A, Gamez L, et al. Adult scoliosis: prevalence, SF-36, and nutritional parameters in an elderly volunteer population. Spine (Phila Pa 1976). 2005;30(9):1082-1085.

2. Robin GC, Span Y, Steinberg R, et al. Scoliosis in the elderly: a follow-up study. Spine (Phila Pa 1976). 1982;7(4):355-359.

3. Zygourakis CC, Liu CY, Keefe M, et al. Analysis of national rates, cost, and sources of cost variation in adult spinal deformity. Neurosurgery. 2018;82(3):378-387.
4. O’Leary PT, Bridwell KH, Lenke LG, et al. Risk factors and outcomes for catastrophic failures at the top of long pedicle screw constructs: a matched cohort analysis performed at a single center. Spine (Phila Pa 1976). 2009;34(20):2134-2139.

5. DeWald CJ, Stanley T. Instrumentation-related complications of multilevel fusions for adult spinal deformity patients over age 65: surgical considerations and treatment options in patients with poor bone quality. Spine (Phila Pa 1976). 2006; 31(19)(suppl):S144-S151.

6. Smith JS, Klineberg E, Lafage V, et al. Prospective multicenter assessment of perioperative and minimum 2-year postoperative complication rates associated with adult spinal deformity surgery. J Neurosurg Spine. 2016;25(1):1-14.

7. Hitchon PW, Brenton MD, Black AG, et al. In vitro biomechanical comparison of pedicle screws, sublaminar hooks, and sublaminar cables. J Neurosurg. 2003;99(1)(suppl):104-109.

8. Murakami H, Yamazaki K, Attallah-Wasif ES, et al. A biomechanical study of 3 different types of sublaminar wire used for constructs in the thoracic spine. J Spinal Disord Tech. 2006;19(6):442-446.

9. Sales de Gauzy J, Jouve JL, Ilharreborde B, et al. Use of the Universal Clamp in adolescent idiopathic scoliosis. Eur Spine J. 2014;23(suppl 4):S446-S451.

10. Gazzeri R, Faiola A, Galarza M, Tamorri M. Universal Clamp system in thoracolumbar spinal fixation: technical note. Acta Neurochir (Wien). 2009;151(12):1673-1680.

11. Roth AK, Boon-Ceelen K, Smelt H, et al. Radiopaque UHMWPE sublaminar cables for spinal deformity correction: Preclinical mechanical and radiopacifier leaching assessment. J Biomed Mater Res B Appl Biomater. 2018; 106(2):771-779. 
12. Hongo M, Ilharreborde B, Gay RE, et al. Biomechanical evaluation of a new fixation device for the thoracic spine. Eur Spine J. 2009;18(8):1213-1219.

13. Hackenberg L, Link T, Liljenqvist U. Axial and tangential fixation strength of pedicle screws versus hooks in the thoracic spine in relation to bone mineral density. Spine (Phila Pa 1976). 2002;27(9):937-942.

14. Coe JD, Warden KE, Herzig MA, McAfee PC. Influence of bone mineral density on the fixation of thoracolumbar implants. A comparative study of transpedicular screws, laminar hooks, and spinous process wires. Spine (Phila Pa 1976). 1990;15(9):902-907.

15. Liljenqvist U, Hackenberg L, Link T, Halm H. Pullout strength of pedicle screws versus pedicle and laminar hooks in the thoracic spine. Acta Orthop Belg. 2001;67(2):157-163.

16. Paxinos O, Tsitsopoulos PP, Zindrick MR, et al. Evaluation of pullout strength and failure mechanism of posterior instrumentation in normal and osteopenic thoracic vertebrae. $J$ Neurosurg Spine. 2010;13(4):469-476.

17. Colman M, Pond J, Bachus K, et al. Fenestrated screws augmented with PMMA increase the pullout strength of sacral pedicle screws. Clin Spine Surg. 2017;30(3):E252-E256.

18. Sun E, Alkalay R, Vader D, Snyder BD. Preventing distal pullout of posterior spine instrumentation in thoracic hyperkyphosis: a biomechanical analysis. J Spinal Disord Tech. 2009;22(4):270-277.

19. Bogie R, Roth AK, de Faber S, et al. Novel radiopaque ultrahigh molecular weight polyethylene sublaminar wires in a growth-guidance system for the treatment of early-onset scoliosis: feasibility in a large animal study. Spine (Phila Pa 1976). 2014:39(25):E1503-E1509.

20. Chong AC, Prohaska DJ, Bye BP. Validation of different combination of three reversing half-hitches alternating posts (RHAPs) effects on arthroscopic knot integrity. Kans J Med. 2017;10(2):35-39.

21. Morgan EF, Bayraktar HH, Keaveny TM. Trabecular bone modulus-density relationships depend on anatomic site. $J$ Biomech. 2003;36(7):897-904.

22. Viswanathan VK, Minnema AJ, Viljoen S, Farhadi HF. Sublaminar banding as an adjunct to pedicle screw-rod constructs: a review and technical note on novel hybrid constructs in spinal deformity surgery. J Neurosurg Spine. 2019; 30(6):807-813.

23. Viswanathan VK, Ganguly R, Minnema AJ, et al. Biomechanical assessment of proximal junctional semi-rigid fixation in long-segment thoracolumbar constructs. J Neurosurg Spine. 2018;30(2):184-192.

24. Cho SK, Caridi J, Kim JS, et al. Attenuation of proximal junctional kyphosis using sublaminar polyester tension bands: a biomechanical study. World Neurosurg. 2018;120: e1136-e1142.

25. Viswanathan VK, Kukreja S, Minnema AJ, Farhadi HF. Prospective assessment of the safety and early outcomes of sublaminar band placement for the prevention of proximal junctional kyphosis. J Neurosurg Spine. 2018;28(5):520-531.

26. Han S, Hyun SJ, Kim KJ, et al. Rod stiffness as a risk factor of proximal junctional kyphosis after adult spinal deformity surgery: comparative study between cobalt chrome multiplerod constructs and titanium alloy two-rod constructs. Spine J. 2017;17(7):962-968.
27. Wirth AJ, Goldhahn J, Flaig C, et al. Implant stability is affected by local bone microstructural quality. Bone. 2011; 49(3):473-478.

28. Wang W, Baran GR, Garg H, et al. The benefits of cement augmentation of pedicle screw fixation are increased in osteoporotic bone: a finite element analysis. Spine Deform. 2014;2(4):248-259.

29. Van den Abbeele M, Valiadis JM, Lima LVPC, et al. Contribution to FE modeling for intraoperative pedicle screw strength prediction. Comput Methods Biomech Biomed Engin. 2018;21(1):13-21.

30. Chevalier Y, Matsuura M, Krüger S, et al. Micro-CT and micro-FE analysis of pedicle screw fixation under different loading conditions. J Biomech. 2018;70:204-211.

31. Carter DR, Hayes WC. Bone compressive strength: the influence of density and strain rate. Science. 1976;194(4270): 1174-1176.

32. Hamasaki T, Tanaka N, Kim J, et al. Pedicle screw augmentation with polyethylene tape: a biomechanical study in the osteoporotic thoracolumbar spine. J Spinal Disord Tech. 2010;23(2):127-132.

\section{Disclosures}

Dr. Arts receives clinical or research support for the study described (includes equipment or materials) from DSM Biomedical. Mr. van Rietbergen is a consultant for Scanco Medical AG.

\section{Author Contributions}

Conception and design: Doodkorte, Belda, Roth, van Rietbergen, Arts, Van Rhijn, Willems. Acquisition of data: Doodkorte. Analysis and interpretation of data: Doodkorte, Belda, Roth, van Rietbergen, Arts. Drafting the article: Doodkorte, Belda, Roth, van Rietbergen. Critically revising the article: Roth, Arts, Lataster, Willems. Reviewed submitted version of manuscript: all authors. Approved the final version of the manuscript on behalf of all authors: Doodkorte. Statistical analysis: Doodkorte. Administrative/technical/material support: Lataster. Study supervision: Roth, Arts, Van Rhijn, Willems.

\section{Supplemental Information}

\section{Previous Presentations}

Poster: Sublaminar tape as alternative and addition to pedicle screws in spinal surgery, 3rd International Workshop on Spine Loading and Deformation, Berlin, Germany, 5 July 2019. Presentation: Sublaminar tape as alternative and addition to pedicle screws in spinal surgery, 25th European Society of Biomechanics, Vienna, Austria, 9 July 2019. Presentation: Pullout testing of spinal instrumentation in an in vivo mimicking experimental setup, European Orthopaedic Research Society, Maastricht, The Netherlands, 3 October 2019.

\section{Correspondence}

Remco Doodkorte: Maastricht University, Maastricht, The Netherlands.rjp.doodkorte@maastrichtuniversity.nl. 